\title{
THE ASYMPTOTIC AGGREGATION PROBLEM AND THE MACRODESCRIPTION OF HIGH DIMENSIONAL SYSTEMS
}

\author{
G.R.ADILOV and G. TINAZTEPE \\ Akdeniz University, Department of Mathematics \\ Antalya, 07058, Turkey \\ E-mail: gabil@akdeniz.edu.tr; gtinaztepe@akdeniz.edu.tr
}

Received September 29, 2004; revised March 30, 2005

\begin{abstract}
In this paper, the aggregation problem for high dimensional systems is studied in a different point of view. The deviations occuring when macro-descriptions are built and evaluated statistically. The systems asymptotic ideal with the aggregation is studied in this sense. Also for some classes of matrices the leading eigenvalues and norms have been taken as aggregate and aggregation is studied in this sense (errors are evaluated statistically).
\end{abstract}

Key words: High dimensional systems, asymptotic aggregation, macro-description, CobbDouglas function, norms and leading eigenvalues of matrices

\section{Introduction}

The high dimensional complicated systems are generally described and studied in terms of macro-parameters. Some reasons for this are the following:

- the complexity of the detailed description,

- the difficulties in solution of the large dimensional systems,

- the insufficiency of the memory of computers.

In addition to these reasons, sometimes in some situations the detailed description is not required. For example, there can exist an exact solution for a large dimensional complicated optimization problem, but the system may not work in an optimal manner.

In economics the systems are usually described via macro-parameters and then studied. The following well-known model can be given as an example. Let $V$ be real output, $K$ the capital stock, and $L$ the labor force. There exists a functional relation $V=f(K, L)$ between them. In reality $V$ depends not only on $K$ and $L$ but also on the distribution of $K$ and $L$. 
Another example is Leontief's static input-output model $x=A x+y$. Here, $x=\left(x_{1}, x_{2}, \ldots, x_{n}\right)$ is the total output vector, $x_{i}$ is the output of $i^{t h}$ industry and it is the sum of the $x_{i}^{k}$ which is the output of the $k^{t h}$ factory. It can be seen that the elements of matrix $A$ are not constant and are dependent on how $x_{i}$ is distributed to the factories.

We can add many similar examples. In general, economic models are based on the parameters which don't take the interior structure of the model into account. There exist differences between macro-description of the model and implementation of the model in practice and these differences must be studied (see [6, 7]).

In dealing with macromodels the aggregation is a very useful tool. Many methods of aggregation have been proposed. Generally systems for which aggregation is applied are classified into two types.

The first ones are the systems in which aggregation is ideal, i.e. the macrodescription of the system can be given without any information loss. This kind of systems are rare and they don't give information about the general situation. The second ones are those in which the aggregation is not ideal. The macro-description of the system is given via required aggregates and the possible information losses are minimized or at least predicted (see [8]).

In $[3,4,5,6,7]$ and in this paper, the aggregation of high dimensional systems is studied in a different viewpoint. The errors arising during transition to macrodescription are evaluated in the sense of mean statistics. This is a different approach from others and many models are examined.

In Section 2, the constant elasticity of substitution production function, which plays important role in economics is considered and for the some special cases of this function concrete expressions of macro-description is derived. Errors existing in transition to macro-description are evaluated in the sense of mean statistics and expressed depending on the dimension of system.It is proved that under certain conditions it goes to zero when dimension goes to infinity.

In Section 3 the leading eigenvalues and norms of matrices are considered as an aggregate for high dimensional matrices. For a specific class of matrices with sufficiently large dimension it is shown that the norms and leading eigenvalues of almost every element of matrix lies in a small neighbourhood of a certain number.

We note that thermodynamic methods are also used for solution of aggregation problems (see $[1,9]$ ). Although the handling of high dimensional systems in our method is similar to that in thermodynamic methods, it differs from the thermodynamic methods in a radical manner.

\section{Asymptotic Aggregation of Constant Elasticity of Substitution Production Function}

The general expression of the constant elasticity of substitution production functions is given in the following form

$$
f\left(x_{1}, x_{2}, \ldots, x_{n}\right)=\left[\sum_{i=1}^{n} \alpha_{i} x_{i}^{-\rho}\right]^{-\frac{\nu}{\rho}},
$$


here $\rho$ denotes the elasticity of production, $\nu$ denotes the degree of homogeneity of the function. Many production functions used in economics are special cases of this function. Here are some examples:

a) If $\sum_{i=1}^{n} \alpha_{i}=1, \alpha_{i} \geq 0, \nu=1$, then we have

$$
\varphi\left(x_{1}, x_{2}, \ldots, x_{n}\right)=\lim _{\rho \rightarrow 0} f\left(x_{1}, x_{2}, \ldots, x_{n}\right)=x_{1}^{\alpha_{1}} x_{2}^{\alpha_{2}} \cdots x_{n}^{\alpha_{n}},
$$

namely the Cobb-Douglas production function;

b) If $\nu=\frac{1}{2}$ and $\rho=-\frac{1}{2}$, then we have

$$
\Psi\left(x_{1}, x_{2}, \ldots, x_{n}\right)=\sum_{i=1}^{n} \alpha_{i} \sqrt{x_{i}},
$$

i.e. the resource distribution function,

c) If $\nu=1$, then we have

$$
\eta\left(x_{1}, x_{2}, \ldots, x_{n}\right)=\lim _{\rho \rightarrow \infty} f\left(x_{1}, x_{2}, \ldots, x_{n}\right)=\min _{i=\overline{1, n}} x_{i},
$$

i.e. the production function with constant ratio. We can add similar examples by changing the given parameters.

In order to show our new idea for the aggregation problem let us take one of the special cases given above, the Cobb-Douglas production function:

$$
\varphi\left(x_{1}, x_{2}, \ldots, x_{n}\right)=x_{1}^{\alpha_{1}} x_{2}^{\alpha_{2}} \cdots x_{n}^{\alpha_{n}} .
$$

When large dimensional systems are studied, the use of the aggregates in the form $X_{i}=\sum_{j=1}^{n} \alpha_{i j} x_{j}$ can be desired for evaluation of Cobb-Douglas production function. For the sake of simplicity, let us consider the following aggregate:

$$
X=\frac{x_{1}+x_{2}+\cdots+x_{n}}{n} .
$$

It seems impossible to evaluate function $\varphi\left(x_{1}, x_{2}, \ldots, x_{n}\right)$ via the parameter $X$ for a general case. But this can be done for large dimension systems. Let us denote the following simplex by $Q(h)$ :

$$
Q(h)=\left\{x=\left(x_{1}, x_{2}, \cdots, x_{n}\right) \mid x_{1}+x_{2}+\cdots+x_{n}=h, x_{i} \geq 0, i=\overline{1, n}\right\} .
$$

It is well-known that the area of this simplex is calculated by the following formula:

$$
S=\int \ldots \int d Q=\frac{\sqrt{n} h^{n-1}}{(n-1) !} .
$$

Let $\bar{\varphi}\left(x_{1}, x_{2}, \ldots, x_{n}\right)$ be the mean value of $\varphi\left(x_{1}, x_{2}, \ldots, x_{n}\right)$ on $Q(h)$ :

$$
\bar{\varphi}\left(x_{1}, x_{2}, \ldots, x_{n}\right)=\frac{1}{S} \iint_{x \in Q} \ldots \int \varphi\left(x_{1}, x_{2}, \ldots, x_{n}\right) d Q .
$$


Theorem 1. Suppose that for $i=\overline{1, n}$ the numbers $\alpha_{i}$ satisfy the following condition

$$
\sum_{i=1}^{n} \alpha_{i}^{2}=o\left(\sum_{i=1}^{n} \alpha_{i}\right)=o(1), n \rightarrow \infty
$$

Then the relative mean quadratic deviation of function $\varphi\left(x_{1}, x_{2}, \ldots, x_{n}\right)$ from $\bar{\varphi}\left(x_{1}, x_{2}, \ldots, x_{n}\right)$ vanishes whenever $n$ goes to infinity, i.e.

$$
\lim _{n \rightarrow \infty} \frac{\frac{1}{S} \int \ldots \int\left(\varphi\left(x_{1}, x_{2}, \ldots, x_{n}\right)-\bar{\varphi}\left(x_{1}, x_{2}, \ldots, x_{n}\right)\right)^{2} d Q}{\bar{\varphi}^{2}\left(x_{1}, x_{2}, \ldots, x_{n}\right)}=0 .
$$

Proof. We can compute the integral of $\varphi\left(x_{1}, x_{2}, \ldots, x_{n}\right)$ on the simplex $Q(h)$ with the help of Dirichlet formula:

$$
\int_{\substack{x_{1}+x_{2}+\cdots+x_{n} \leq 1 \\ x_{1}, \ldots, x_{n} \geq 0}} x_{1}^{p_{1}-1} \cdots x_{n}^{p_{n}-1} d x_{1} \cdots d x_{n}=\frac{\Gamma\left(p_{1}\right) \cdots \Gamma\left(p_{n}\right)}{\Gamma\left(p_{1}+\cdots+p_{n}+1\right)} .
$$

Then we find

$$
\int_{x \in Q} \ldots \int \varphi\left(x_{1}, x_{2}, \ldots, x_{n}\right) d Q=\frac{\sqrt{n} h^{n} A_{n}}{n !}
$$

where

$$
A_{n}=\Gamma\left(\alpha_{1}+1\right) \Gamma\left(\alpha_{2}+1\right) \cdots \Gamma\left(\alpha_{n}+1\right)
$$

So we can write

$$
\bar{\varphi}\left(x_{1}, x_{2}, \ldots, x_{n}\right)=\frac{A_{n} h}{n} .
$$

Now let us compute the mean quadratic distance of $\varphi\left(x_{1}, x_{2}, \ldots, x_{n}\right)$ from $\bar{\varphi}\left(x_{1}, x_{2}, \ldots, x_{n}\right)$ :

$$
\begin{aligned}
& \frac{1}{S} \int_{x \in Q} \ldots \int_{x \in Q}\left(\varphi\left(x_{1}, x_{2}, \ldots, x_{n}\right)-\bar{\varphi}\left(x_{1}, x_{2}, \ldots, x_{n}\right)\right)^{2} d Q \\
& \quad=\frac{1}{S} \int_{x \in Q} \ldots \int x_{1}^{2 \alpha_{1}} \ldots x_{n}^{2 \alpha_{n}} d Q-\frac{A_{n}^{2} h^{2}}{n^{2}}=\left\{\frac{\tilde{A}_{n}}{n(n+1)}-\frac{A_{n}^{2}}{n^{2}}\right\} h^{2},
\end{aligned}
$$

where

$$
\tilde{A}_{n}=\Gamma\left(1+2 \alpha_{1}\right) \Gamma\left(1+2 \alpha_{2}\right) \cdots \Gamma\left(1+2 \alpha_{n}\right) .
$$

The relative mean quadratic distance can be calculated via (2.2) and (2.3):

$$
d(n) \equiv \frac{\frac{1}{S} \int_{x \in Q} \ldots \int\left(\varphi\left(x_{1}, x_{2}, \ldots, x_{n}\right)-\bar{\varphi}\left(x_{1}, x_{2}, \ldots, x_{n}\right)\right)^{2} d Q}{\bar{\varphi}^{2}\left(x_{1}, x_{2}, \ldots, x_{n}\right)}=\frac{n}{n+1} \frac{\tilde{A}_{n}}{A_{n}^{2}}-1 .
$$

Writing the Taylor expansions of $\ln \tilde{A}_{n}$ and $\ln A_{n}^{2}$, the following equalities are obtained 


$$
\begin{aligned}
& \ln \tilde{A}_{n}=\sum_{i=1}^{n} \ln \Gamma\left(1+2 \alpha_{i}\right)=\sum_{i=1}^{n} \ln \Gamma(1)+\frac{\Gamma^{\prime}(1)}{\Gamma(1)} \sum_{i=1}^{n} 2 \alpha_{i}+O\left(\sum_{i=1}^{n} 4 \alpha_{i}^{2}\right), \\
& \ln A_{n}^{2}=2 \sum_{i=1}^{n} \ln \Gamma\left(1+\alpha_{i}\right)=2 \sum_{i=1}^{n} \ln \Gamma(1)+2 \frac{\Gamma^{\prime}(1)}{\Gamma(1)} \sum_{i=1}^{n} \alpha_{i}+O\left(\sum_{i=1}^{n} \alpha_{i}^{2}\right) .
\end{aligned}
$$

Taking into account condition (2.1) and equalities

$$
\sum_{i=1}^{n} \alpha_{i}=1, \quad \Gamma(1)=1, \quad \Gamma^{\prime}(1)=-c,
$$

we obtain

$$
\ln A_{n}=-2 c+o(1), \quad \ln A_{n}^{2}=-2 c+o(1),
$$

where $c$ is the Euler constant ( $c=0.577215 \ldots$.). Referring (2.4) and the statements given above it follows that

$$
\lim _{n \rightarrow \infty} d(n)=\lim _{n \rightarrow \infty}\left\{\frac{n}{n+1} \frac{\tilde{A}_{n}}{A_{n}^{2}}-1\right\}=0 .
$$

The proof is completed.

Remark 1. If condition (2.1) is provided, then it can be easily shown that $\lim _{n \rightarrow \infty} A_{n}=$ $e^{-c}$. Considering this observation and Theorem 1 , we can prove that for sufficiently large $n$ function $\varphi\left(x_{1}, x_{2}, \ldots, x_{n}\right)$ can be expressed via $X$ aggregate in the following form (note, that for sufficiently large $n$ the mean statistics error $d(n)$ is close enough to zero):

$$
\varphi\left(x_{1}, x_{2}, \ldots, x_{n}\right) \approx e^{-c} X .
$$

Repeating the same arguments for the aggregate $\tilde{X}=\sqrt{\frac{1}{n}\left(x_{1}^{2}+x_{2}^{2}+\ldots+x_{n}^{2}\right)}$, a similar theorem can be proved and the following result can be obtained:

$$
\varphi\left(x_{1}, x_{2}, \ldots, x_{n}\right) \approx \frac{e^{-\frac{c}{2}}}{\sqrt{2}} \tilde{X}
$$

See $[5,7]$ for details.

Remark 2. Many special cases of constant elasticity of substitution production function are studied in a similar way (see [7]), but no theoretical results are obtained for a general case. The following formula, which is confirmed by numerical experiments and is compatible with the results of special cases, is asserted: for sufficiently large $n$ the equality

$$
f\left(x_{1}, x_{2}, \ldots, x_{n}\right) \approx \nu \Gamma(\nu) \sum_{i=1}^{n} \alpha_{i} X^{q}
$$

is valid, where $q=\max \{1+\rho,-\rho\}$ and $\alpha_{i}$ satisfies some necessary conditions. 
Remark 3. In studying hierarchical optimization problems, the aggregation of the subsystems by using this approach is a very necessary step [6,7].

Remark 4. The obtained results show that the idea "Having large dimension makes the study of system difficult every time " is not true, i.e., macro-description of system can be given with less error as dimension is increased.

\section{The Leading Eigenvalues and Norms of High Dimensional Matrices}

In this section we investigate the other types of problems by using the same approach. It is well-known that the norm and the leading eigenvalue of matrix is characterized as a necessary aggregate macro-parameter of the given matrix.

Let us investigate these parameters in the class of high dimensional matrices with non-negative arrays. This class of matrices is often used in mathematical economics, probability theory, small oscillations theory of elastic systems and etc. Let $M(n)$ be a set of square matrices of order $n$, and the following sets be defined as above;

$$
\begin{aligned}
& K(n ; h)=\left\{A \in M(n): 0 \leq a_{i j} \leq h, i, j=1, n\right\}, \\
& G(n ; h, \varepsilon, \mu)=\left\{A \in K(n ; h):\left|\frac{\lambda(A)}{n}-\mu\right| \leq \varepsilon\right\}, \\
& \Phi(n ; h, \varepsilon, \mu)=\left\{A \in K(n ; h):\left|\frac{\|A\|}{n}-\mu\right| \leq \varepsilon\right\}
\end{aligned}
$$

where $\lambda(A)$ is the leading eigen-value of the matrix $A ; h, \varepsilon, \mu$ are the given numbers satisfying the following conditions $\mu>\varepsilon>0, \mu+\varepsilon \leq h$.

Theorem 2. Let $\varepsilon>0$ be a given number as small as desired. Then

$$
\begin{gathered}
{\left[1-\frac{h^{4}}{\varepsilon^{4}}\left(\frac{1}{48 n^{2}}-\frac{1}{120 n^{3}}\right)\right]^{n} \theta} \\
\quad \leq \frac{\text { meas } G(n ; h, \varepsilon, \mu)}{\operatorname{meas} K(n ; h)} \leq 1, \\
{\left[1-\frac{h^{4}}{\varepsilon^{4}}\left(\frac{1}{48 n^{2}}-\frac{1}{120 n^{3}}\right)\right]^{n} \theta\left(1-\frac{h^{4}}{\varepsilon^{4}}\left(\frac{1}{48 n^{2}}-\frac{1}{120 n^{3}}\right)\right)} \\
\leq \frac{\text { meas } \Phi(n ; h, \varepsilon, \mu)}{\text { meas } K(n ; h)} \leq 1,
\end{gathered}
$$

for $\mu=\frac{h}{2}$, where $\theta(x)$ is a Heavyside function:

$$
\theta(x)= \begin{cases}1, & \text { if } x>0 \\ 0, & \text { if } x \leq 0\end{cases}
$$


Proof. The following inequalities hold for non-negative matrices:

$$
\min _{i} \sum_{j=1}^{n} a_{i j} \leq \lambda(A) \leq \max _{i} \sum_{j=1}^{n} a_{i j}=\|A\| .
$$

Let us denote $\Lambda(n ; h, \varepsilon, \mu)=\left\{A \in K(n ; h):\left|\frac{1}{n} \sum_{j=1}^{n} a_{i j}-\mu\right| \leq \varepsilon, \quad i=\overline{1, n}\right\}$. Then it is clear that

$$
\begin{aligned}
& \Lambda(n ; h, \varepsilon, \mu) \subset \Phi(n ; h, \varepsilon, \mu) \subset K(n ; h), \\
& \Lambda(n ; h, \varepsilon, \mu) \subset G(n ; h, \varepsilon, \mu) \subset K(n ; h),
\end{aligned}
$$

and it is sufficient to show that

$$
\begin{aligned}
{\left[1-\frac{h^{4}}{\varepsilon^{4}}\left(\frac{1}{48 n^{2}}-\frac{1}{120 n^{3}}\right)\right]^{n} } & \theta\left(1-\frac{h^{4}}{\varepsilon^{4}}\left(\frac{1}{48 n^{2}}-\frac{1}{120 n^{3}}\right)\right) \\
\leq & \frac{\text { meas } \Lambda(n ; h, \varepsilon, \mu)}{\text { meas } K(n ; h)} \leq 1
\end{aligned}
$$

for $\mu=\frac{h}{2}$. Let us calculate meas $\Lambda(n ; h, \varepsilon, \mu)$. Since $a_{i j}(i, j=\overline{1, n})$ are independent variables, we get

$$
\begin{aligned}
& \operatorname{meas} \Lambda(n ; h, \varepsilon, \mu)=\overbrace{\int \ldots \int \quad}^{n^{2}} d a_{11} d a_{12} \ldots d a_{n n} \\
& \left|\frac{1}{n} \sum_{\substack{j=1 \\
0 \leq a_{i j} \leq h, \quad}}^{n} a_{i j}-\mu\right| \leq \varepsilon, i=\overline{1, n} \\
& =\left[\int \ldots \int d a_{11} d a_{12} \ldots d a_{1 n}\right]^{n}=\left[h^{n}-\int \ldots \int d a_{11} d a_{12} \ldots d a_{1 n}\right]^{n} . \\
& \mid \begin{array}{ll}
\frac{1}{n} \sum_{j=1}^{n} a_{1 j}-\mu \mid \leq \varepsilon, & \mid \begin{array}{l}
\frac{1}{n} \sum_{j=1}^{n} a_{1 j}-\mu \mid>\varepsilon, \\
0 \leq a_{1 j} \leq h, \quad j=\overline{1, n}
\end{array} \\
0 \leq a_{1 j} \leq h, \quad j=\overline{1, n}
\end{array} \\
& \mid \begin{array}{l}
\frac{1}{n} \sum_{j=1}^{n} a_{1 j}-\mu \mid>\varepsilon \\
0 \leq a_{1 j} \leq h, j=\overline{1, n}
\end{array}
\end{aligned}
$$
written as follows:

$$
\left\{a_{1 j}:\left(\frac{1}{n} \sum_{j=1}^{n} a_{1 j}-\mu\right)^{4}>\varepsilon^{4}, 0 \leq a_{1 j} \leq h, j=\overline{1, n}\right\}
$$


thus

$$
V(n ; h, \varepsilon, \mu)=\overbrace{\int \ldots \int_{\left(\frac{1}{n} \sum_{j=1}^{n} a_{1 j}-\mu\right)^{4}>\varepsilon^{4},}^{n}}^{n} d a_{11} d a_{12} \ldots d a_{1 n} .
$$

Also we have the following inequality:

$$
\int_{0}^{h} \ldots \int_{0}^{h}\left(\frac{1}{n} \sum_{j=1}^{n} a_{1 j}-\mu\right)^{4} d a_{11} d a_{12} \ldots d a_{1 n} \geq \varepsilon^{4} \underset{\substack{\left.\frac{1}{n} \sum_{j=1}^{n} a_{1 j}-\mu\right)^{4}>\varepsilon^{4}, 0 \leq a_{1 j} \leq h, \quad, \quad j=\overline{1, n}}}{\int} d a_{11} d a_{12} \ldots d a_{1 n} .
$$

Hence it follows that

$$
V(n ; h, \varepsilon, \mu) \leq \frac{1}{\varepsilon^{4}} \int_{0}^{h} \ldots \int_{0}^{h}\left(\frac{1}{n} \sum_{j=1}^{n} a_{1 j}-\mu\right)^{4} d a_{11} d a_{12} \ldots d a_{1 n} .
$$

Now let us calculate the integral at the right-hand side of this inequality

$$
\begin{aligned}
\int_{0}^{h} \ldots & \int_{0}^{h}\left(\frac{1}{n} \sum_{j=1}^{n} a_{1 j}-\mu\right)^{4} d a_{11} d a_{12} \ldots d a_{1 n} \\
= & \frac{1}{n^{4}} \int_{0}^{h} \ldots \int_{0}^{h}\left[\left(\sum_{j=1}^{n} a_{1 j}\right)^{4}-4 \mu n\left(\sum_{j=1}^{n} a_{1 j}\right)^{3}+6 \mu^{2} n^{2}\left(\sum_{j=1}^{n} a_{1 j}\right)^{2}\right. \\
& \left.-4 \mu^{3} n^{3}\left(\sum_{j=1}^{n} a_{1 j}\right)+\mu^{4} n^{4}\right] d a_{11} d a_{12} \ldots d a_{1 n} \\
\int_{0}^{h} \ldots & \int_{0}^{h}\left(\sum_{j=1}^{n} a_{1 j}^{4} d a_{11} d a_{12} \ldots d a_{1 n}\right. \\
& =\int_{0}^{h} \ldots \int_{0}^{h}\left(\sum_{i \neq j \neq k \neq l}^{n} a_{1 j} a_{1 i} a_{1 k} a_{1 l}+6 \sum_{i \neq j \neq k}^{n} a_{1 j}^{2} a_{1 i} a_{1 k}\right. \\
& \left.+3 \sum_{i \neq j}^{n} a_{1 i}^{2} a_{1 j}^{2}+4 \sum_{i \neq j}^{n} a_{1 j}^{3} a_{1 i}+\sum_{j=1}^{n} a_{1 j}^{4}\right) d a_{11} d a_{12} \ldots d a_{1 n}
\end{aligned}
$$




$$
\begin{aligned}
= & n(n-1)(n-2)(n-3) \frac{h^{n+4}}{16}+6 n(n-1)(n-2) \frac{h^{n+4}}{12} \\
& +3 n(n-1) \frac{h^{n+4}}{8}+4 n(n-1) \frac{h^{n+4}}{8}+n \frac{h^{n+4}}{5} \\
= & h^{n+4}\left(\frac{n(n-1)(n-2)(n-3)}{16}+\frac{n(n-1)(n-2)}{2}\right. \\
& \left.+\frac{5 n(n-1)}{6}+\frac{n}{5}\right) .
\end{aligned}
$$

Analogously, we have

$$
\begin{aligned}
& \int_{0}^{h} \ldots \int_{0}^{h}\left(\sum_{j=1}^{n} a_{1 j}\right)^{3} d a_{11} d a_{12} \ldots d a_{1 n}=h^{n+3}\left(\frac{n(n-1)(n+2)}{8}+\frac{n}{4}\right) \\
& \int_{0}^{h} \ldots \int_{0}^{h}\left(\sum_{j=1}^{n} a_{1 j}\right)^{2} d a_{11} d a_{12} \ldots d a_{1 n}=h^{n+2}\left(\frac{n(n-1)}{4}+\frac{n}{3}\right), \\
& \int_{0}^{h} \ldots \int_{0}^{h}\left(\sum_{j=1}^{n} a_{1 j}\right) d a_{11} d a_{12} \ldots d a_{1 n}=h^{n+1} \frac{n}{2} .
\end{aligned}
$$

Substituting (3.4) - (3.7) into (3.3), we get the following equality

$$
\begin{aligned}
& \int_{0}^{h} \ldots \int_{0}^{h}\left(\frac{1}{n} \sum_{j=1}^{n} a_{1 j}-\mu\right)^{4} d a_{11} d a_{12} \ldots d a_{1 n} \\
& \quad=h^{4}\left[\left(\mu-\frac{h}{2}\right)^{4}+\left(\mu-\frac{h}{2}\right)^{2} \frac{h^{2}}{2 n}+\frac{h^{4}}{48 n^{2}}-\frac{h^{4}}{120 n^{3}}\right] .
\end{aligned}
$$

Thus we have the estimate of $V$ :

$$
V(n ; h, \varepsilon, \mu) \leq \frac{h^{4}}{\varepsilon^{4}}\left[\left(\mu-\frac{h}{2}\right)^{4}+\left(\mu-\frac{h}{2}\right)^{2} \frac{h^{2}}{2 n}+\frac{h^{4}}{48 n^{2}}-\frac{h^{4}}{120 n^{3}}\right]
$$

and the following inequality

$$
\begin{aligned}
& \text { meas } \Lambda(n ; h, \varepsilon, \mu) \\
& \geq h^{n^{2}}\left\{1-\frac{1}{\varepsilon^{4}}\left[\left(\mu-\frac{h}{2}\right)^{4}+\left(\mu-\frac{h}{2}\right)^{2} \frac{h^{2}}{2 n}+\frac{h^{4}}{48 n^{2}}-\frac{h^{4}}{120 n^{3}}\right]\right\}^{n} \\
& \times \theta\left[1-\frac{1}{\varepsilon^{4}}\left(\left(\mu-\frac{h}{2}\right)^{4}+\left(\mu-\frac{h}{2}\right)^{2} \frac{h^{2}}{2 n}+\frac{h^{4}}{48 n^{2}}-\frac{h^{4}}{120 n^{3}}\right)\right] .
\end{aligned}
$$

The expression at the right hand side achieves its maximum (with respect to the parameter $\mu$ ) for $\mu=\frac{h}{2}$. Thus (3.1) follows from (3.8). The theorem is proved. 
The following corollary is an immediate result of Theorem 2 .

Corollary 1.

$$
\lim _{n \rightarrow \infty} \frac{\text { meas } G(n ; h, \varepsilon, \mu)}{\text { meas } K(n ; h)}=\lim _{n \rightarrow \infty} \frac{\text { meas } \Phi(n ; h, \varepsilon, \mu)}{\text { meas } K(n ; h)}=1 \text { for } \mu=\frac{h}{2} .
$$

Remark 5. If for the estimation of $V(n ; h, \varepsilon, \mu)$ instead of (3.2) we would take the domain

$$
\left\{a_{1 j}:\left(\frac{1}{n} \sum_{j=1}^{n} a_{1 j}-\mu\right)^{2}>\varepsilon^{2}, 0 \leq a_{1 j} \leq h, j=\overline{1, n}\right\},
$$

then this estimate would be rough and we can't obtain the desired result, since

$$
\int_{0}^{h} \ldots \int_{0}^{h}\left(\frac{1}{n} \sum_{j=1}^{n} a_{1 j}-\mu\right)^{2} d a_{11} d a_{12} \ldots d a_{1 n}=h^{n}\left[\left(\mu-\frac{h}{2}\right)^{2}+\frac{h^{2}}{12 n}\right]
$$

and then

$$
\begin{array}{r}
\left\{1-\frac{1}{\varepsilon^{2}}\left[\left(\mu-\frac{h}{2}\right)^{2}+\frac{h^{2}}{12 n}\right]\right\}^{n} \theta\left[1-\frac{1}{\varepsilon^{2}}\left(\left(\mu-\frac{h}{2}\right)^{2}+\frac{h^{2}}{12 n}\right)\right] \\
\leq \frac{\operatorname{meas} \Phi(n ; h, \varepsilon, \mu)}{\operatorname{meas} K(n ; h)} \leq 1 .
\end{array}
$$

For $\mu=\frac{h}{2}$, the left hand side is equal to

$$
\left(1-\frac{h^{2}}{12 n \varepsilon^{2}}\right)^{n} \theta\left(1-\frac{h^{2}}{12 n \varepsilon^{2}}\right)
$$

which converges to $e^{-\frac{h^{2}}{12 \varepsilon^{2}}}$ for $n \rightarrow \infty$.

As it follows from the proof of Theorem 2, the part of this theorem related to norms is valid for all matrices, i.e. the constraint about positivity of elements is not necessary.

Useful results can be obtained by applying Theorem 2 to the problems which include norms and leading eigenvalues of matrices, some examples are presented in [2].

\section{Conclusions}

In this article, a macro-description problem (i.e. a description of the system by using less variables) is studied in a different viewpoint. It is an effective way to examine the 
complex systems with high dimensions and interesting results are derived. The errors existing in transition to macro-description are evaluated in the sense of mean statistics. It is a different approach from others and we have shown that the approximation error goes to zero when dimension of the system goes to infinity.

The special cases of the constant elasticity of substitution production function, for example, the Cobb-Douglas production function, are investigated and concrete expressions for macro-description are derived. In transition to macro-description, the errors are derived in the sense of mean statistics and they are evaluated with respect to dimension. It is shown that these errors go to zero when the dimension goes to infinity.

Then the norms and the largest eigenvalues of matrices which play important role in linear systems are studied. It is shown that when the dimensions of matrices in a certain class are increased infinitely, the measure of matrices whose norms and smallest eigenvalues are out of the neighborhood of a certain point goes to zero.

\section{References}

[1] Adilov G.R. Estimate of adequacy by using entropy methods for investigation of socialeconomic systems. In: Collection of works "Mechanisms of control for the socialeconomic system”, $54-58,1988$.

[2] Adilov G.R. On convergence speed of iterative process of systems of linear equations on large dimensions. In: Proceedings of the Institute of Math. And Mech. Acad. Sci. of Azerbaijan, number 9, 7-9, 1998.

[3] Adilov G.R. On norm and leading eigenvalues of non-negative high-dimension matrices. In: Proceedings of the Institute of Math. And Mech. Acad. Sci. of Azerbaijan, volume 11, $347-393,1999$.

[4] Adilov G.R. On the mathematical modelling of high dimensional systems. In: XV. Turkish National Mathematical Symposium, volume 1. Mersin, 2002.

[5] Adilov G.R. and Tinaztepe R. A new approach to the problem of modelling the systems with high dimension. In: International Scientifi c Conference, Chernivtsi, Ukraine, 2003.

[6] Adilov G.R. and Opoitsev V.I. Aggregation of linear systems. Automatika \& Telemekhanika, 8, $64-72,1986$.

[7] Adilov G.R. and Opoitsev V.I. On asymptotic aggregation. Automatika \& Telemekhanika, 4(1), 131 - 140, 1989.

[8] A. Pervozvansky and V. Gaitsgory. Decomposition, Agreggation and Suboptization. Nauka, Moscow, 1979.

[9] A.G. Wilson. Entropy in Urban and Regional Analysis. Nauka Publishing, Moscow, 1979. 
Asimptotinis agregacijos uždavinys ir didelès eilès sistemų makrodeskripcija

G.R. Adilov, G. Tinaztepe

Straipsnyje nagrinejjamas daugiamačių sistemų agregacijos uždavinys. Nuokrypiai, atsirandantys keičiant sistemą jos makrodeskripcija, ịvertinti statistiškai. Sistemos agreguotasis asimptotinis idealas nagrinėjamas tuo pačiu požiūriu. Taip pat straipsnyje nagrinėjama kelių matricų klasių agregacija, ju pagrindines tikrines vertes ir normas naudojant kaip agregatą ir nuokrypius įvertinant statistiškai. 